\title{
Diagnostic Accuracy of Screening MR Imaging Using Unenhanced Axial CISS and Coronal T2WI for Detection of Small Internal Auditory Canal Lesions
}

\author{
T.A. Abele, D.A. Besachio, E.P. Quigley, R.K. Gurgel, C. Shelton, H.R. Harnsberger, and R.H. Wiggins III
}

\begin{abstract}
BACKGROUND AND PURPOSE: While enhanced TIWI is considered the "gold standard" for detection of internal auditory canal pathology, unenhanced fluid-sensitive sequences have shown high sensitivity for lesion identification. Our purpose was to evaluate the diagnostic accuracy of an unenhanced MR imaging protocol using axial CISS and coronal T2WI for detection of small (10 mm or less) internal auditory canal lesions.
\end{abstract}

\begin{abstract}
MATERIALS AND METHODS: Twenty-three patients with small internal auditory canal lesions and 13 patients without lesions who had undergone MR imaging using the screening protocol and confirmatory gadolinium-enhanced thin section TIWI were identified. Two blinded neuroradiologists retrospectively evaluated all examinations using 1) only axial CISS, 2) only coronal T2WI, and 3) axial and coronal sequences together. Accuracy, specificity, sensitivity, and interobserver agreement were assessed.

RESULTS: Median maximum lesion dimension was $4 \mathrm{~mm}$ (range, 2-10 mm). Accuracy, specificity, and sensitivity for axial CISS alone were $0.94,0.96$, and 0.91 for observer 1 and $0.94,0.92$, and 1.00 for observer 2 . The data for the coronal T2WI sequence only were $0.94,0.96$, and 0.91 for observer 1 , and $0.99,1.00$, and 0.96 for observer 2 . Using axial and coronal sequences, the data were $0.97,0.96$, and 1.00 for observer 1 , and $0.99,0.98$, and 1.00 for observer 2 . $\kappa$ coefficients were 0.84 for the axial sequence only, 0.90 for coronal only, and 0.91 for axial and coronal both.
\end{abstract}

CONCLUSIONS: Screening noncontrast MR imaging using a combination of axial CISS and coronal T2WI sequences can detect small internal auditory canal lesions with $100 \%$ sensitivity and excellent interobserver agreement.

ABBREVIATION: IAC = internal auditory canal

V estibular schwannoma is the most common lesion diagnosed during MR imaging evaluation of unilateral sensorineural hearing loss. ${ }^{1,2}$ Nevertheless, only $2.7 \%-4.7 \%$ of contrast-enhanced MRIs performed for audiovestibular symptoms will diagnose vestibular schwannomas., ${ }^{3,4}$ Although gadolinium-enhanced thin section MR imaging has historically been considered the "gold standard" for detection of internal auditory canal (IAC) tumors such as vestibular schwannomas, lower cost unenhanced, fluid-sensitive sequences have demonstrated pooled sensitivities

Received March 10, 2014; accepted after revision May 24.

From the Departments of Radiology (T.A.A., E.P.Q., H.R.H., R.H.W.), Biomedical Informatics (R.H.W.), and Division of Otolaryngology-Head and Neck Surgery (R.K.G., C.S., R.H.W.), University of Utah, Salt Lake City, Utah; and Department of Radiology (D.A.B.), Naval Medical Center Portsmouth, Portsmouth, Virginia. Paper previously presented at: Annual Meeting of the American Society of Head and Neck Radiology, September 26, 2013; Milwaukee, Wisconsin.

Please address correspondence to Travis A. Abele, MD, Department of Radiology, University of Utah, 30 North 1900 East \#1A071, Salt Lake City, UT 84132; e-mail: travis.abele@hsc.utah.edu

http://dx.doi.org/10.3174/ajnr.A4041 ranging from $96 \%$ to $98 \%$ for detection of IAC lesions ranging from $2 \mathrm{~mm}$ to $>20 \mathrm{~mm}$ in diameter. ${ }^{5}$ In the era of rising health care costs, especially for diagnostic imaging, the cost savings associated with a low-cost screening IAC MR imaging may become an important factor in decision-making.

At our institution, we have performed screening MR imaging of the IACs since the 1990s using fluid-sensitive axial and coronal sequences. In 1996, Allen et $\mathrm{al}^{6}$ demonstrated $98 \%$ accuracy of an axial and coronal T2-weighted IAC screening MR imaging protocol in 25 patients whose lesions had a mean diameter of $12 \mathrm{~mm}$. Two lesions measuring $<5 \mathrm{~mm}$ were missed. In 2006, the axial T2 FSE sequence at our institution was replaced by an axial dualexcitation balanced steady-state interference sequence termed CISS (Siemens, Erlangen, Germany), as 3D CISS had been reported to have twice the contrast-to-noise ratio compared with 3D T2WI. ${ }^{7}$ As no study has evaluated the accuracy of a 2-plane screening IAC MR imaging protocol using CISS for detection of small ( $\leq 10 \mathrm{~mm}$ ) lesions, we chose to evaluate the diagnostic accuracy of a 2-sequence screening MR imaging protocol 
using unenhanced axial CISS and coronal T2WI for detection of small IAC lesions.

\section{MATERIALS AND METHODS}

The study was approved by the institutional review board and was compliant with the Health Insurance Portability and Accountability Act. Our institutional radiologic information system database was reviewed from February 2006 to April 2013 for patients who had undergone both 1) the unenhanced screening IAC MR imaging protocol and 2) the contrast-enhanced thin section T1weighted MR imaging of the IACs. Patients were included if their examinations were within 13 months of each other or the contrast-enhanced MR imaging was performed after the screening MR imaging. The cases with IAC lesions $>10 \mathrm{~mm}$, prior surgery, and/or inadequate diagnostic quality secondary to motion or other artifacts were then excluded. Two lesions located in the cochlea that were readily visible on the noncontrast screening MR imaging were excluded because they were few in number and because this study was focused on IAC lesions.

The remaining cases, including 23 patients with IAC lesions and 13 patients without lesions, were then reviewed by 2 Certificate of Added Qualification neuroradiologists who were blinded to the original interpretations. One observer was an assistant professor with 5 years of neuroradiology experience after fellowship, and the other observer was a professor of neuroradiology with 14 years of experience. Each observer retrospectively evaluated both IACs 3 separate times from the screening IAC MR imaging study. First, only the axial CISS sequence was reviewed for all IACs, then only the coronal T2 sequence was reviewed, and then both sequences were reviewed together. At least a 24-hour interval and study rerandomization was performed between each review to decrease recollection bias. Observers were asked to identify if there was a lesion within the IAC that warranted further evaluation with contrast-enhanced MR imaging. The absence of pathology was determined when both the vestibulocochlear and facial nerves were visualized from their root entry/exit zones to the fundus of the IAC without adjacent mass. Observers were also asked to identify the location of the lesions as being: 1) intracanalicular (located within the IAC without contacting the fundus of the IAC), or 2) fundal (within the IAC with any portion of the lesion in contact with the fundus). All examinations were interpreted on a diagnostic PACS workstation during a normal work day in between clinical examinations to simulate a routine study interpretation by the neuroradiologists. All answers were recorded by a third party. After all reading sessions of the studies, the noncontrast interpretations were compared with the correlating postcontrast studies to confirm the presence or absence of a lesion. The greatest lesion diameter in either the transverse, anteroposterior, or craniocaudal dimension was measured on contrast-enhanced MR imaging as part of the inclusion criteria.

All MRIs were performed on a $1.5 \mathrm{~T}$ or $3 \mathrm{~T}$ MR imaging scanner. With a few exceptions, all axial 3D CISS sequences were performed with the following parameters: TR, 5.91-7.85; TE, 2.963.93; averages, $1-2$; flip angle, $37^{\circ}-80^{\circ}$; and voxel size, $0.3 \times 0.3 \times$ $0.8 \mathrm{~mm}$ or larger. With a few exceptions, coronal 3D T2 sequences were performed with the following parameters: TR, 750; TE, $110-$ 15 ; averages, 1 ; flip angle, $170^{\circ}$; and voxel size, $0.3 \times 0.3 \times 1.24$
Table 1: Diagnostic data for observer 1

\begin{tabular}{lccc}
\hline & Axial CISS & Coronal T2WI & CISS + T2WI \\
\hline Accuracy & 0.94 & 0.94 & 0.97 \\
Specificity & $0.96(0.85-0.99)$ & $0.96(0.85-0.99)$ & $0.96(0.85-0.99)$ \\
Sensitivity & $0.91(0.70-0.98)$ & $0.91(0.70-0.94)$ & $1.00(0.82-1.00)$ \\
\hline Note:-All data are percentage $(95 \% \mathrm{Cl})$.
\end{tabular}

Table 2: Diagnostic data for observer 2

\begin{tabular}{lccc}
\hline & Axial CISS & Coronal T2WI & CISS + T2WI \\
\hline Accuracy & 0.94 & 0.99 & 0.99 \\
Specificity & $0.92(0.80-0.97)$ & $1.00(0.94-1.00)$ & $0.98(0.88-1.00)$ \\
Sensitivity & $1.00(0.82-1.00)$ & $0.96(0.76-1.00)$ & $1.00(0.82-1.00)$ \\
\hline
\end{tabular}

Note:-All data are percentage $(95 \% \mathrm{Cl})$.

$\mathrm{mm}$ or larger. The standard body coil was used to transmit radiofrequency pulses, and a 16-channel head coil was used to receive signal in all patients. In general, the axial 3D CISS sequence was 2 minutes 45 seconds to 4 minutes 30 seconds in acquisition time and the coronal 3D T2 sequence was 4 minutes 35 seconds to 4 minutes 59 seconds in acquisition time. Including the 3 plane localizers, the average total scan time was 10 minutes 2 seconds to 10 minutes 43 seconds.

Statistical analysis included assessment of sensitivity, specificity, and accuracy of the axial CISS sequence only, the coronal T2 sequence only, and both sequences together, using the T1 postgadolinium correlating studies as a "gold standard." Interobserver agreement was assessed with $\kappa$ coefficient analysis.

\section{RESULTS}

Of 865 screening IAC examinations performed between February 2006 and April 2013, 36 cases had both the screening MR imaging and the postcontrast MR imaging that met the inclusion criteria. These included 23 patients with radiologically identified IAC lesions measuring $\leq 10 \mathrm{~mm}$ in greatest diameter and 13 control patients without lesions on contrast-enhanced T1-weighted MR imaging. Thus, a total of 72 IACs were included: 23 with a lesion and 49 without pathology. The ages of patients with an IAC lesion ranged from 29 to 81 years (median 65 years); 12 patients were women and 11 were men. The patients without pathology were considered the control group and ranged in age from 36 to 85 years (median 53 years). Six were women and 7 were men.

Fourteen lesions were intracanalicular and 9 were fundal in location. The lesion size ranged from 2 to $10 \mathrm{~mm}$ in greatest diameter, the mean greatest diameter was $4.7 \mathrm{~mm}$, and the median greatest diameter was $4 \mathrm{~mm}$. Four of 23 lesions were resected. Three were vestibular schwannomas, and 1 was a ganglioneuroma. One lesion was treated with radiation therapy, and the others were followed and thus had no histologic evaluation.

Accuracy, specificity, and sensitivity were calculated for both observers using the axial CISS alone, coronal T2WI alone, and both sequences together, and are detailed in Tables 1 and 2. Observer 1 achieved less than $100 \%$ sensitivity with either sequence alone, but did achieve $100 \%$ sensitivity when evaluating the 2 sequences together. Observer 2 performed with $100 \%$ sensitivity using the axial CISS sequence alone and with both sequences together. The 2 lesions undetected by observer 1 on the axial sequence alone were at the fundus of the IAC and measured 3 and 4 $\mathrm{mm}$ in maximum diameter. These lesions were more conspicuous on the coronal sequences (Fig 1). On the coronal sequence alone, 

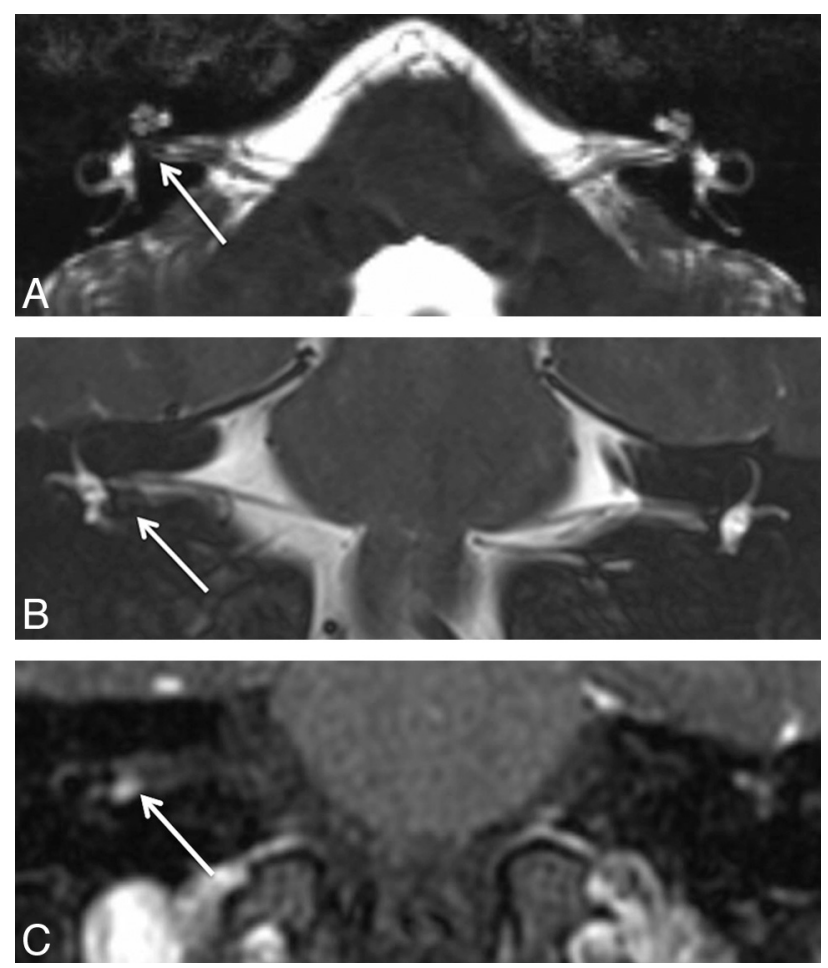

FIG 1. A 29-year-old man with hearing loss on the right. A, Axial CISS image shows subtle asymmetric hypointense signal within the fundus of the right IAC (arrow), which could be dismissed as volume averaging. B, Coronal T2WI better demonstrates the hypointense lesion (arrow) along the inferior right IAC fundus, which is confirmed to be a 4-mm enhancing mass (arrow) on postcontrast coronal TTWI (C).

observers 1 and 2 missed the same fundal IAC lesion and observer 1 missed an additional fundal lesion. These lesions were more apparent on the axial sequences (Fig 2).

Observer 1 had 2 false-positives on the axial sequence and 2 false-positives on the coronal sequence, whereas observer 2 had 4 false-positives on the axial and 0 false-positives on the coronal sequence. Using both sequences, observer 1 had 2 false-positives and observer 2 had 1 false-positive, which was a different patient from observer 1 . On retrospective review, the 3 false-positives generated on evaluation of both sequences were felt to be secondary to volume averaging from the adjacent wall of the IAC, banding artifact on the axial CISS that was not resolved on the coronal T2WI, and volume averaging from the anterior inferior cerebellar artery within the IAC (Fig 3).

$\kappa$ coefficients for interobserver reliability were 0.84 for the axial CISS alone and 0.90 for coronal T2WI alone. For both sequences together, the $\kappa$ coefficient was 0.91 .

\section{DISCUSSION}

In this study, we showed that a 2-sequence screening MR imaging protocol using axial CISS and coronal T2WI can reliably detect IAC lesions with 100\% sensitivity, high accuracy and specificity, and excellent interobserver reliability. Screening diagnostic tools are intended to have a high sensitivity. The high sensitivity, however, creates a small number of false-positives, which may necessitate confirmatory imaging with contrast administration.

Early detection of vestibular schwannomas and other IAC lesions is important to reduce treatment morbidity because larger
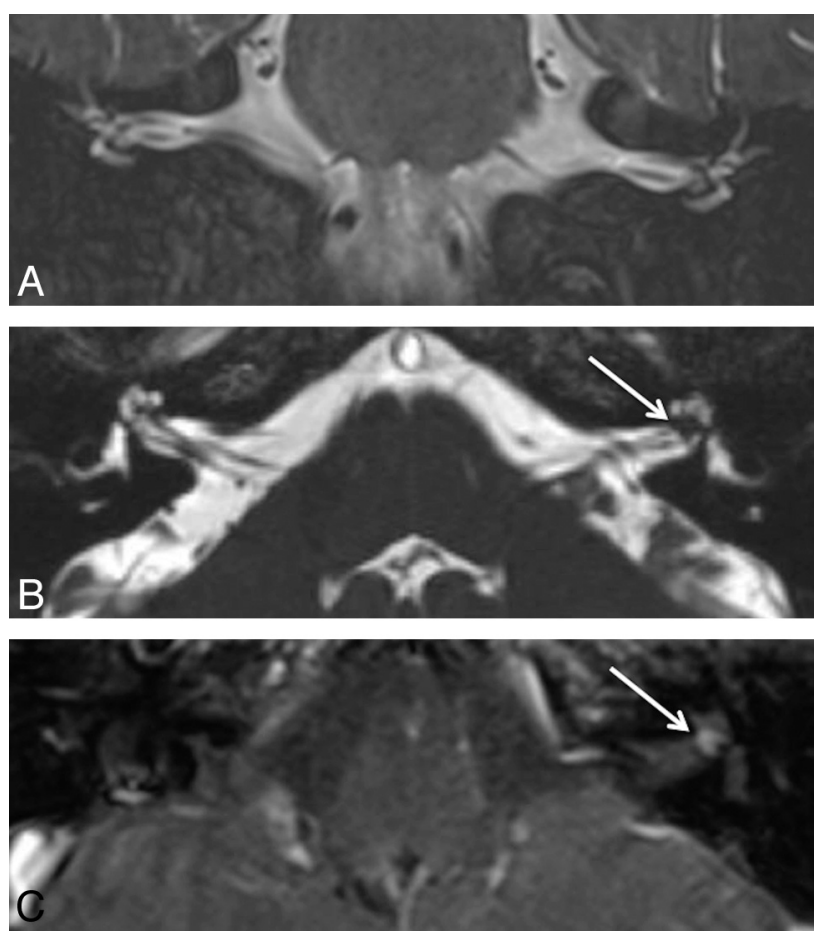

FIG 2. A 65-year-old woman who presented with left-sided hearing loss. A, Coronal T2WI shows the intracanalicular bilateral vestibulocochlear and facial nerves without associated lesion. B, Axial CISS image demonstrates a hypointense lesion (arrow) to better advantage located within the fundus of the left IAC. C, Postcontrast axial TIWI confirms the enhancing 2-mm lesion (arrow).

tumors cause more treatment-related complications, particularly with regard to facial nerve outcomes. ${ }^{8}$ Early detection is particularly important if surgery is attempted to preserve hearing; this is because the size of the tumor correlates with hearing outcomes. ${ }^{9,10}$ As the trend moves to less audiometric testing and more initial imaging evaluation, a smaller percentage of IAC MRIs will be positive for vestibular schwannoma. While gadolinium-enhanced MR imaging is considered a standard and embraced by many, in the era of rising health care costs, adoption of a less expensive and less time intensive screening protocol is warranted. This study proves that unenhanced MR imaging can detect even small subcentimeter IAC lesions, which have been considered by many to require gadolinium administration for detection.

The measure of a screening examination is its ability to detect mild or early forms of disease, which for this study translates into recognition of very small IAC lesions. In the late 1990s and early 2000s, at least 8 studies ${ }^{3,4,6,11-15}$ evaluated the diagnostic value of unenhanced fluid-sensitive MR imaging sequences (T2WI or CISS) for the evaluation of IAC lesions. Five of those studies reported the number of lesions measuring $\leq 10 \mathrm{~mm} .{ }^{6,11-14}$ Of the 5, 2 had $100 \%$ sensitivity using axial 2D fast spin-echo T2WI alone and axial 3D fast asymmetric spin-echo T2WI alone and included 10 and 11 patients, respectively. ${ }^{12,14}$ Of the remaining 3 studies, Allen et $\mathrm{al}^{6}$ included 12 small lesions, in which 2 lesions (measuring less than $4 \mathrm{~mm}$ ) were missed by 2 of 4 observers in small internal auditory canals using axial and coronal $\mathrm{T} 2$ fast spin-echo with voxel size of approximately $0.3 \times 0.4 \times 3 \mathrm{~mm}$. Stuckey et $\mathrm{al}^{11}$ evaluated 4 lesions measuring $\leq 10 \mathrm{~mm}$ in which a 4 - $\mathrm{mm}$ IAC 

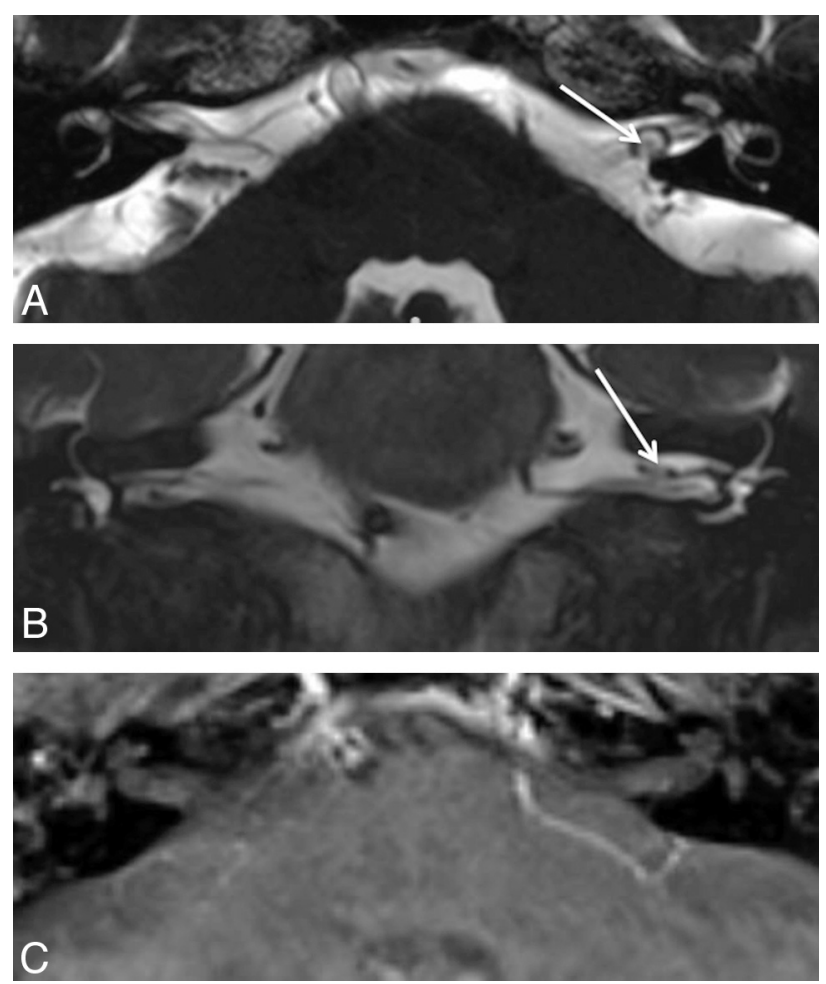

FIG 3. A 71-year-old man with vertigo. A, Axial CISS image demonstrates a hypointense focus surrounded by a loop of the anterior inferior cerebellar artery, which was thought to represent a small IAC lesion. $B$, Coronal T2WI shows the same hypointense focus. $C$, Postcontrast axial TIWI shows no enhancing lesion, indicating that the hypointense focus on the screening study was volume averaging related to the adjacent artery.

lesion was undetected by 1 of 2 observers on axial 3D CISS with voxel size of approximately $0.7 \mathrm{~mm}$ isotropic. Hermans et $\mathrm{al}^{13}$ imaged 9 small lesions in which a 3-mm IAC lesion was missed by both observers, and a 6-mm intralabyrinthine lesion was missed by 1 observer using a $0.7 \times 0.7 \times 3.0 \mathrm{~mm}$ axial 3D CISS sequence.

Comparing our data to prior studies supports a diagnostic benefit of the 2 plane, 2-sequence screening protocol using coronal T2WI and axial CISS. Two planes mitigate the effects of artifact from volume averaging, which can be especially limiting in small IACs. In the Hermans et $\mathrm{al}^{13}$ study, both observers attributed misses and uncertainty primarily to volume averaging, but also to artifacts and suboptimal techniques. In the Allen et $\mathrm{al}^{6}$ publication, 2 lesions measuring 2 and $3 \mathrm{~mm}$ in maximum diameter were missed likely because of volume averaging because they were specifically noted to be found in small IACs. ${ }^{6}$

Another advantage of the 2-sequence protocol is the benefit of superior contrast-to-noise ratio provided by CISS imaging in addition to having the T2 sequence to mitigate the banding artifact, which may limit balanced steady-state free precession sequences such as CISS. The 3D CISS imaging has twice the contrast-tonoise ratio for the intracanalicular nerves and CSF compared with the $3 \mathrm{D}$ fast recovery fast spin-echo T2WI sequence, ${ }^{7}$ which is likely why it has replaced T2WI for evaluation of the IACs in many institutions. However, CISS sequences are limited by banding artifact resulting from magnetic field inhomogeneity, ${ }^{16}$ which can lead to the appearance of pseudolesions in the evaluation of the
IACs. T2WI can be used to confirm or exclude suspected lesions on CISS that may be artifactual.

Unenhanced screening sequences provide significant cost and time savings compared with gadolinium-enhanced IAC MR imaging. The institutional charge of our screening IAC protocol is $\$ 850$ less than our contrast-enhanced IAC MR imaging protocol, which is due to a lack of gadolinium contrast, reduced scan time, and reduced professional fee. The screening protocol takes approximately 11 minutes versus 30 minutes for the contrast-enhanced examination. Over 1 year from April 2012 to April 2013, 127 screening IAC studies were performed for asymmetric hearing loss at our institution. Of those, 101 were normal, 20 were performed to follow already diagnosed IAC or intralabyrinthine lesions, 5 had incidental lesions unrelated to hearing symptoms, and 1 study had an IAC lesion that will be further characterized by a contrast-enhanced MR imaging. Using the screening protocol rather than the contrast-enhanced IAC protocol over that year, we saved at least $\$ 107,100(126 \times \$ 850)$, which is a slight overestimate because it does not account for the redundant screening examination performed on the 1 patient with an IAC lesion. Three studies ${ }^{6,17,18}$ demonstrated similar cost saving per examination with the difference in unenhanced and enhanced protocols equaling \$550-\$800. MR imaging time savings over that same year equal approximately 2394 minutes $(126 \times 19$ minutes $)$ or 99.75 hours of MR imaging scan time.

A 2009 meta-analysis ${ }^{5}$ supported by the United Kingdom National Institute for Health Research reviewed over 11 studies from 1996 to 2001 assessing the diagnostic accuracy and cost-effectiveness of noncontrast $\mathrm{T} 2$ or $\mathrm{T} 2{ }^{\star}$ sequences in the identification of vestibular schwannoma. Fortnum et $\mathrm{al}^{5}$ found that fluid-sensitive sequences have pooled sensitivities ranging from $96 \%-98 \%$ for detection of IAC lesions measuring $2 \mathrm{~mm}$ to $>20 \mathrm{~mm}$ in diameter. They concluded that noncontrast fluid-sensitive MR imaging sequences allow for accurate evaluation of the facial and vestibulocochlear nerves within the cerebellopontine angle and IAC, and that including contrast-enhanced sequences is less cost effective and not likely to add information that would change management in a screening population.

While unenhanced screening MR imaging is sufficient to exclude lesions of the IAC, noncontrast-enhanced MR imaging could potentially miss some pathology of the IAC or labyrinth that would be detected with contrast. As Jackler ${ }^{19}$ addressed in his response to the screening studies published in the late 1990s, noncontrast screening MR imaging may not reliably detect labyrinthitis, vestibular neuritis, sarcoidosis, or leptomeningeal metastases. In the case of the first 2 diagnoses, however, treatment is based on clinical symptoms, not radiologic appearance. For patients who present with symptoms of acute vertigo with or without sudden sensorineural hearing loss, the optimal treatment will be a short course of high-dose steroids, regardless of the imaging findings. ${ }^{20,21}$ In the setting of suspected sarcoidosis or primary malignancy, a contrast-enhanced examination is warranted rather than a screening examination. Furthermore, sarcoidosis or leptomeningeal metastases rarely present as isolated sensorineural hearing loss. Clinical judgment on the part of the ordering clinician is still required to select the best examination and to determine when a screening scan is appropriate. The unenhanced screening MR im-

AJNR Am J Neuroradiol 35:2366-70 Dec 2014 www.ajnr.org 
aging protocol is meant for patients with audiovestibular symptoms without other comorbidities that might affect the IAC or inner ear.

Limitations of this study include its small sample size, few controls, retrospective nature, and lack of pathologic confirmation in most cases. The small sample size was unavoidable given the rare incidence of IAC lesions in the population, especially lesions measuring $\leq 10 \mathrm{~mm}$ in diameter. Similarly, a prospective study of this sample size would be difficult given the time necessary to recruit patients with such a rare lesion. Few controls were available because the unenhanced screening examination was almost exclusively used during the surveyed time span. Patients who had a normal noncontrast screening examination rarely underwent a "gold standard" contrast-enhanced examination and vice versa. Pathologic confirmation could not be obtained in most of the cases because the lesions were small enough and lacking in symptoms to permit follow-up rather than surgery.

Lastly, it could be argued that similar sensitivity data would not be achieved with general radiologists as observers rather than experienced neuroradiologists. We contend that although there may be a brief learning curve, a general radiologist can just as easily trace unaffected vestibulocochlear nerves or detect an asymmetric hypointense mass on a background of hyperintense CSF in the IAC as a Certificate of Added Qualification-holding neuroradiologist.

\section{CONCLUSIONS}

Screening MR imaging using a combination of unenhanced axial CISS and coronal T2WI sequences can reliably detect small $(\leq 10$ $\mathrm{mm}$ ) IAC schwannomas with $100 \%$ sensitivity and excellent interobserver reliability. The high sensitivity creates a small number of false-positives, which may necessitate confirmatory imaging with contrast administration. Fundal lesions may be missed on an axial or coronal sequence alone because of volume averaging.

Disclosures: Clough Shelton-UNRELATED: Grants/Grants Pending: Sonitus,* Cochlear Corp, ${ }^{*}$ Comments: Research into bone conduction hearing aid (Sonitus); research into cochlear implants (Cochlear Corp). H. Ric Harnsberger-UNRELATED: Board Membership: Amirsys, Comments: Work has no relationship to peer-reviewed articles; Employment: Amirsys, Comments: Work has no relationship to peerreviewed articles; Stock/Stock Options: Amirsys, Comments: Work has no relationship to peer-reviewed articles. *Money paid to the institution.

\section{REFERENCES}

1. Kumar A, Maudelonde C, Mafee M. Unilateral sensorineural hearing loss. Laryngoscope 1986;96:14-18

2. Armington WG, Harnsberger HR, Smoker WR, et al. Normal and diseased acoustic pathway: evaluation with MR imaging. Radiology 1988;167:509-15

3. Zealley IA, Cooper RC, Clifford KM, et al. MRI screening for acoustic neuroma: a comparison of fast spin echo and contrast enhanced imaging in 1233 patients. Br J Radiol 2000;73:242-47

4. Annesley-Williams DJ, Laitt RD, Jenkins JP, et al. Magnetic reso- nance imaging in the investigation of sensorineural hearing loss: is contrast enhancement still necessary? J Laryngol Otol 2001;115:14-21

5. Fortnum H, O'Neill C, Taylor R, et al. The role of magnetic resonance imaging in the identification of suspected acoustic neuroma: a systematic review of clinical and cost effectiveness and natural history. Health Technol Assess 2009;13:iii-iv, ix-xi, 1-154

6. Allen RW, Harnsberger HR, Shelton C, et al. Low-cost high-resolution fast spin-echo MR of acoustic schwannoma: an alternative to enhanced conventional spin-echo MR? AJNR Am J Neuroradiol 1996;17:1205-10

7. Lane JI, Ward H, Witte RJ, et al. 3-T imaging of the cochlear nerve and labyrinth in cochlear-implant candidates: $3 \mathrm{D}$ fast recovery fast spin-echo versus $3 \mathrm{D}$ constructive interference in the steady state techniques. AJNR Am J Neuroradiol 2004;25:618-22

8. Gurgel RK, Dogru S, Amdur RL, et al. Facial nerve outcomes after surgery for large vestibular schwannomas: do surgical approach and extent of resection matter? Neurosurg Focus 2012;33:E16

9. Yates PD, Jackler RK, Satar B, et al. Is it worthwhile to attempt hearing preservation in larger acoustic neuromas? Otol Neurotol 2003;24:460-64

10. Meyer TA, Canty PA, Wilkinson EP, et al. Small acoustic neuromas: surgical outcomes versus observation or radiation. Otol Neurotol 2006;27:380-92

11. Stuckey SL, Harris AJ, Mannolini SM. Detection of acoustic schwannoma: use of constructive interference in the steady state three-dimensional MR. AJNR Am J Neuroradiol 1996;17:1219-25

12. Soulié D, Cordoliani YS, Vignaud J, et al. MR imaging of acoustic neuroma with high resolution fast spin echo T2-weighted sequence. Eur J Radiol 1997;24:61-65

13. Hermans R, Van der Goten A, De Foer B, et al. MRI screening for acoustic neuroma without gadolinium: value of 3DFT-CISS sequence. Neuroradiology 1997;39:593-98

14. Naganawa S, Ito T, Fukatsu H, et al. MR imaging of the inner ear: comparison of a three-dimensional fast spin-echo sequence with use of a dedicated quadrature-surface coil with a gadolinium-enhanced spoiled gradient-recalled sequence. Radiology 1998;208: 679-85

15. Marx SV, Langman AW, Crane RC. Accuracy of fast spin echo magnetic resonance imaging in the diagnosis of vestibular schwannoma. Am J Otolaryngol 1999;20:211-16

16. Bangerter NK, Hargreaves BA, Vasanawala SS, et al. Analysis of multiple-acquisition SSFP. Magn Reson Med 2004;51:1038-47

17. Daniels RL, Shelton C, Harnsberger HR. Ultra high resolution nonenhanced fast spin echo magnetic resonance imaging: cost-effective screening for acoustic neuroma in patients with sudden sensorineural hearing loss. Otolaryngol Head Neck Surg 1998;119:364-69

18. Tan TY. Non-contrast high resolution fast spin echo magnetic resonance imaging of acoustic schwannoma. Singapore Med J 1999;40:27-31

19. Jackler RK. Cost-effective screening for acoustic neuroma with unenhanced MR: a clinician's perspective. AJNR Am J Neuroradiol 1996;17:1226-28

20. Strupp M, Zingler VC, Arbusow V, et al. Methylprednisolone, valacyclovir, or the combination for vestibular neuritis. $N$ Engl J Med 2004;351:354-61

21. Rauch SD, Halpin CF, Antonelli PJ, et al. Oral vs intratympanic corticosteroid therapy for idiopathic sudden sensorineural hearing loss: a randomized trial. JAMA 2011;305:2071-79 